\title{
La Cattedrale dell'Elettricità: trasformazione del rudere, permanenza dell'immagine. Il caso della Battersea Power Station di Londra
}

\author{
DOSSIER PATRIMONIO DELL'ELETTRICITÀ: CITTÀ E PAESAGGI ELETTRICI
}

\section{Emanuele Morezzi}

Arch. Ph.D. DAD - Dipartimento di Architettura e Design, Politecnico di Torino. Torino [Piemonte] Italy <emanuele.morezzi@polito.it>.

\section{Riassunto}

Le recenti trasformazioni della Battersea Power Station impongono una riflessione sulla modifica del paesaggio industriale londinese del XX secolo. L'articolo intende porre una riflessione non solo sulla conservazione/trasformazione del rudere, ma anche sulla valenza iconica dell'edificio e sull'importanza del simbolo che esso rappresenta nel panorama londinese, in qualità di caso studio di architettura legata al periodo industriale e alle architetture per la produzione di energia elettrica.

\section{Parole chiave}

Battersea. Centrale elettrica. Rudere. Trasformazione. Restauro. Immagine.

The Cathedral of Electricity: transformation of the ruin, permanence of the image. The case of Battersea Power Station, London

\begin{abstract}
The transformations of the Battersea Power Station impose a reflection on the modification of the London industrial landscape of the twentieth century. The article is focused not only on the preservation / transformation of the power station's ruins, but also on the iconic value of the building and the importance of the symbol which it represents in the London landscape, as a case study of architecture related to industrial architectures.
\end{abstract}

\section{Keywords}

Battersea. Power plant. Ruin. Transformation. Restoration. Image. 


\section{Introduzione}

Nell'analisi delle trasformazioni urbane e architettoniche, nelle modifiche che hanno interessato e che interessano edifici o quartieri, riusciamo a ricostruire la storia dell'intera città o area metropolitana. Studiare non solo gli effetti dei cambiamenti in un edificio, ma anche e soprattutto le cause che li hanno determinati, può aiutare a ricostruire le dinamiche dello sviluppo di un intero centro urbano o di una intera società. Ciò è ancora più evidente relazionandoci alla tematica, ancora aperta, della conservazione e riuso/rifunzionalizazione dell'archeologia industriale del XIX e XX secolo, ed in particolar modo delle centrali elettriche, dove un recente passato costruttivo ha fatto coesistere, gli uni a fianco agli altri, edifici residenziali e produttivi, creando un nuovo modo di intendere la città, associando ampie aree produttive ai centri storicizzati. Negli ultimi decenni, la mutazione economica e produttiva ha però restituito una pesante eredità, costituita spesso dall'abbandono di grandi esempi del recente passato industriale, a cui non si è stati in grado di dare un nuovo futuro che sapesse rispettare le valenze storiche del bene e le nuove necessità delle città. Tale presupposto, riguardante moltissime realtà europee, genera spesso una situazione di sospensione delle decisioni, in cui le antiche architetture industriali, prive di alcuna funzione, sono condannate a diventare ruderi dimenticati e avulsi da ogni piano di conservazione o trasformazione, e a rappresentare iconicamente la fine del periodo industriale che le ha prodotte ${ }^{1}$ (Horne, 2012).

Un caso studio di particolare interesse per sondare questa tendenza, appare essere la Battersea Power Station di Londra, recentemente al centro di una serie di importanti trasformazioni funzionali e costruttive. L'edificio, però, a differenza di molte realtà analoghe, ha nella propria storia di

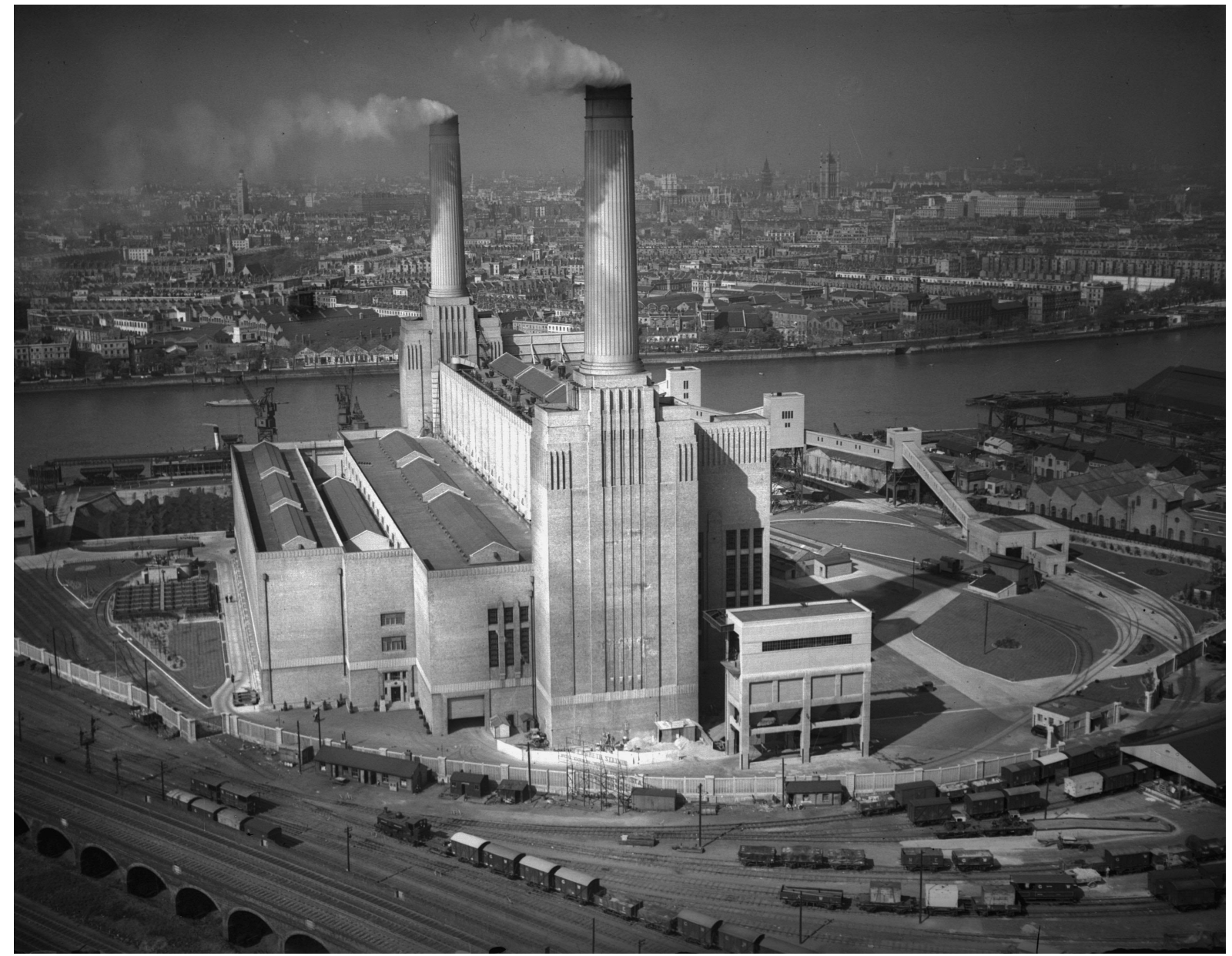

Figura 1. Immagine aerea della centrale in costruzione, completa solo per metà. Anno 1935 circa.

1 Fra la vastissima bibliografia di riferimento si segnalano i testi squisitamente dedicati al patrimonio industriale londinese: M. Horne, London area supply: a survey of London electric lighting and power stations, 2012; K. Sabbagh, Power into art: creating the Tate modern, Bankside, Penguin Gropu, 2000; E. Stirling, The history of the gas light \& Coke company 1812-1949, A.\&C. Black, 1992; Eletric Council, Elettricity supply in the UK: A Chronology, 1987. 
meno di un secolo, tutte le caratteristiche per rappresentare in modo paradigmatico la vita stessa della capitale inglese, riuscendo, come vedremo, a rappresentare in modo estremamente veritiero non solo l'atteggiamento pubblico nei confronti dell'archeologia industriale, ma anche le fasi storiche che la città e l'intera nazione inglese hanno vissuto lungo il XX secolo. L'analisi storica delle vicende della fabbrica, ricca di alterne fasi di prestigio e abbandono, consentirà quindi di meglio comprendere le ultime trasformazioni in atto, il cui progetto definitivo è stato approvato meno di 2 anni fa e che verrà ultimato, secondo le previsioni, nel 2020.

Se, quindi, le varie fasi passate della fabbrica possono illuminarci per meglio comprendere la storia della città e, più in generale del patrimonio industriale inglese, allora è lecito supporre che l'avanguardistico piano di intervento sulla fabbrica possa meglio spiegarci le dinamiche della Londra del futuro e il destino che molte architetture industriali londinesi subiranno nei prossimi anni, diventando da simboli iconici del passato industriale, nuove aree di speculazione e di investimento per capitali stranieri, desiderosi di capitalizzare la superficie costruttiva a disposizione. Da questa analisi, principalmente basata su di una scansione cronologica, delle trasformazioni reali o tentate della Battersea Power Station, si intende creare un parallelo per mettere a fuoco le trasformazioni dell'intera città di Londra, allo scopo di valutare i rischi che il patrimonio culturale e architettonico dovrà affrontare nel futuro.

\section{Premesse e costruzione della cattedrale dell'elettricità}

L'ideazione di ciò che sarà la Battersea Power Station (BPS) nasce dalla necessità di provvedere alla crescente domanda di energia elettrica della Londra del XX secolo e dal bisogno di aggiornare un impianto elettrico cittadino che appariva antiquato 2 (Pedroche, 2013).

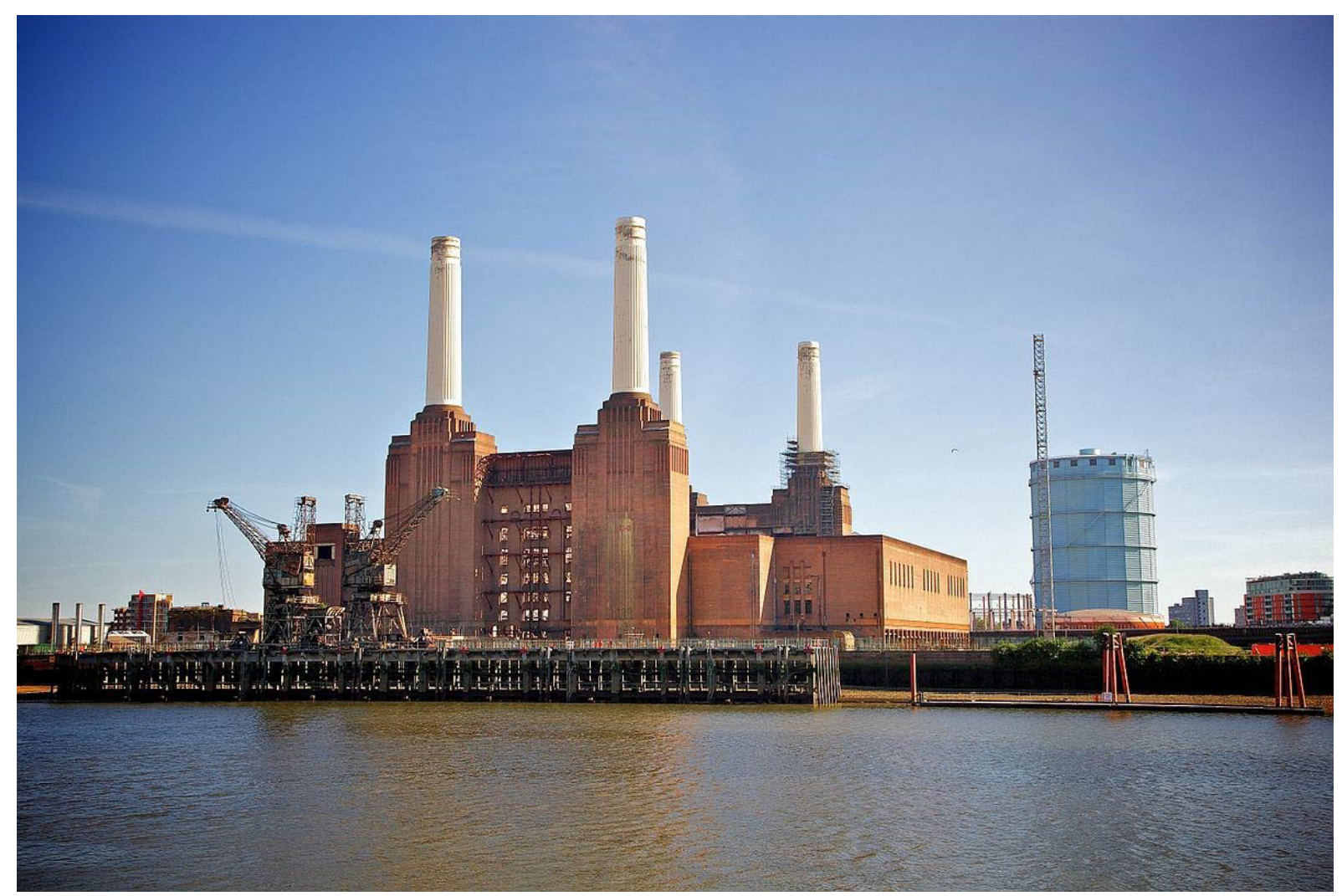

Figura 2. La BPS a metà degli anni 00: un bene abbandonato.

\footnotetext{
2 Le prime centrali elettriche nacquero con lo scopo di rifornire di energia le loro immediate vicinanze. Coprire una maggiore distanza risultava impossibile per via delle dispersioni lungo la tratta. Da questo aspetto, dalla metà del XIX secolo, Londra si popola di una fitta rete di edifici, spesso analoghi dimensionalmente e architettonicamente. Per approfondimenti si veda: Ben Pedroche, London's lost Power Stations and Gasworks, The History press, 2013, cap. I-III
} 
L'eredità degli ultimi anni del periodo vittoriano aveva infatti permesso la realizzazione di un sistema di fornitura dell'energia elettrica che si basava essenzialmente su di una serie di piccole centrali indipendenti, che provvedevano alla fornitura dell'area circostante, normalmente inferiore alla dimensione di quartiere 3 (Watts, 2016). Tale configurazione, che si era venuta a creare nella seconda metà del XIX secolo, risultava non più adeguata alle richieste della Londra degli anni Venti e quindi, anche grazie all'Eletric Lightning Act"4 la società leader del settore propose alla municipalità la costruzione di una nuova centrale. Questo nuovo progetto era caratterizzato dall'enorme ambizione e dalla volontà di creare un edificio senza precedenti nel panorama inglese o europeo: costruire la più grande centrale elettrica del Paese nel pieno centro della capitale. Inoltre l'edificio, per la sua importanza dimensionale e la sua centralità presupponeva un nuovo linguaggio architettonico e compositivo tale da celebrare l'importanza della struttura e della sua funzione. Gli investimenti alla base del progetto Battersea, furono di natura privata, sicuramente incoraggiati dall'Eletric Lighting Act di cui si è detto. Fu appositamente costituita una nuova compagnia, la London Power Company (LPC) 5 .

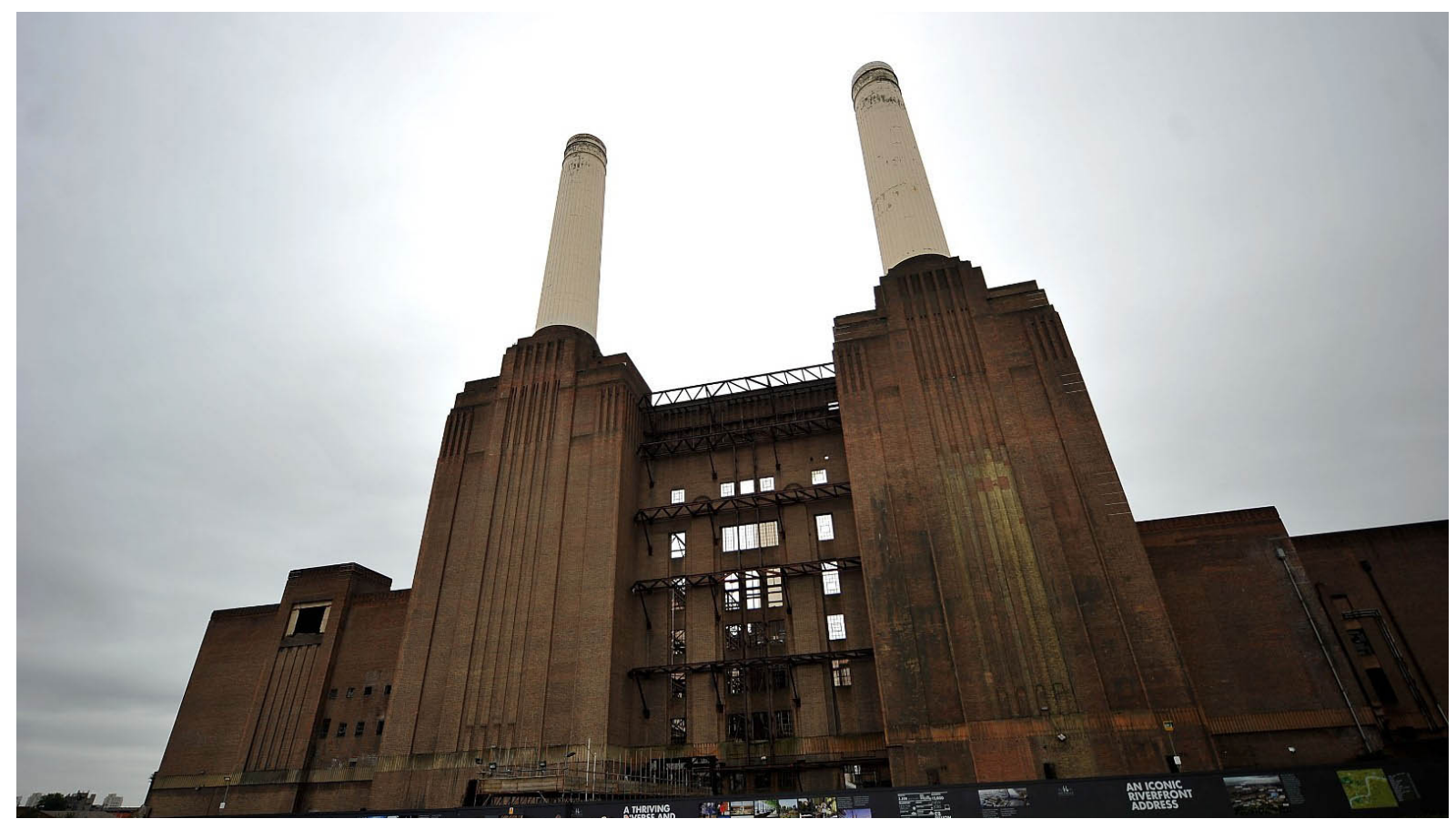

Figura 3. Il prospetto Nord della centrale. I muri perimetrali sono solo uno scheletro senza funzione.

In questo periodo molte centrali appartenenti alla LPC vennero rinnovate e rese maggiormente funzionali e nuovi siti vennero presi in considerazione per la costruzione di complessi innovativi. L'area della futura costruzione era estremamente centrale nel piano di sviluppo di Londra e ciò consentiva di poter rifornire le aree adiacenti la centrale senza la posa di lunghi cavi: grazie alla sua posizione, avrebbe potuto costituire l'approvvigionamento elettrico del centro cittadino, l'area in cui la domanda di energia elettrica era maggiore. Inoltre, la vicinanza con il fiume e con la Victoria Station la rendevano facilmente accessibile ai rifornimenti di carbone sia dal fiume che dalla terraferma. In tal senso, l'idea della LPC di costruire la più grande centrale elettrica del mondo nel cuore di Londra appariva di difficile approvazione da parte del governo cittadino e da

\footnotetext{
${ }_{3}^{3}$ L'esempio più rappresentativo risulta essere la centrale di Deptford, situata nel cuore di Londra e vero paradigma per la costruzione di Battersea, di pochi anni successiva. P. Watts, Up in smoke. The failed dreams of Battersea power station, Paradise Road, London, 2016, p. 31.

${ }^{4}$ L'Eletric Lightining Act del 1882 è stato un provvedimento del governo inglese teso a favorire l'investimento privato nel settore della produzione di energia elettrica, ma regolamentando nello stesso tempo, in modo preciso lo scavo per la posa di cavi e infrastrutture.

${ }^{5}$ La LPC fu creata inglobando ben 10 piccole aziende operanti nel settore, ciascuna proprietaria di piccole centrali elettriche costruite negli anni precedenti. L'importanza della nuova società sul mercato fu enorme, tanto da divenire presto leader cittadino nel settore.
} 


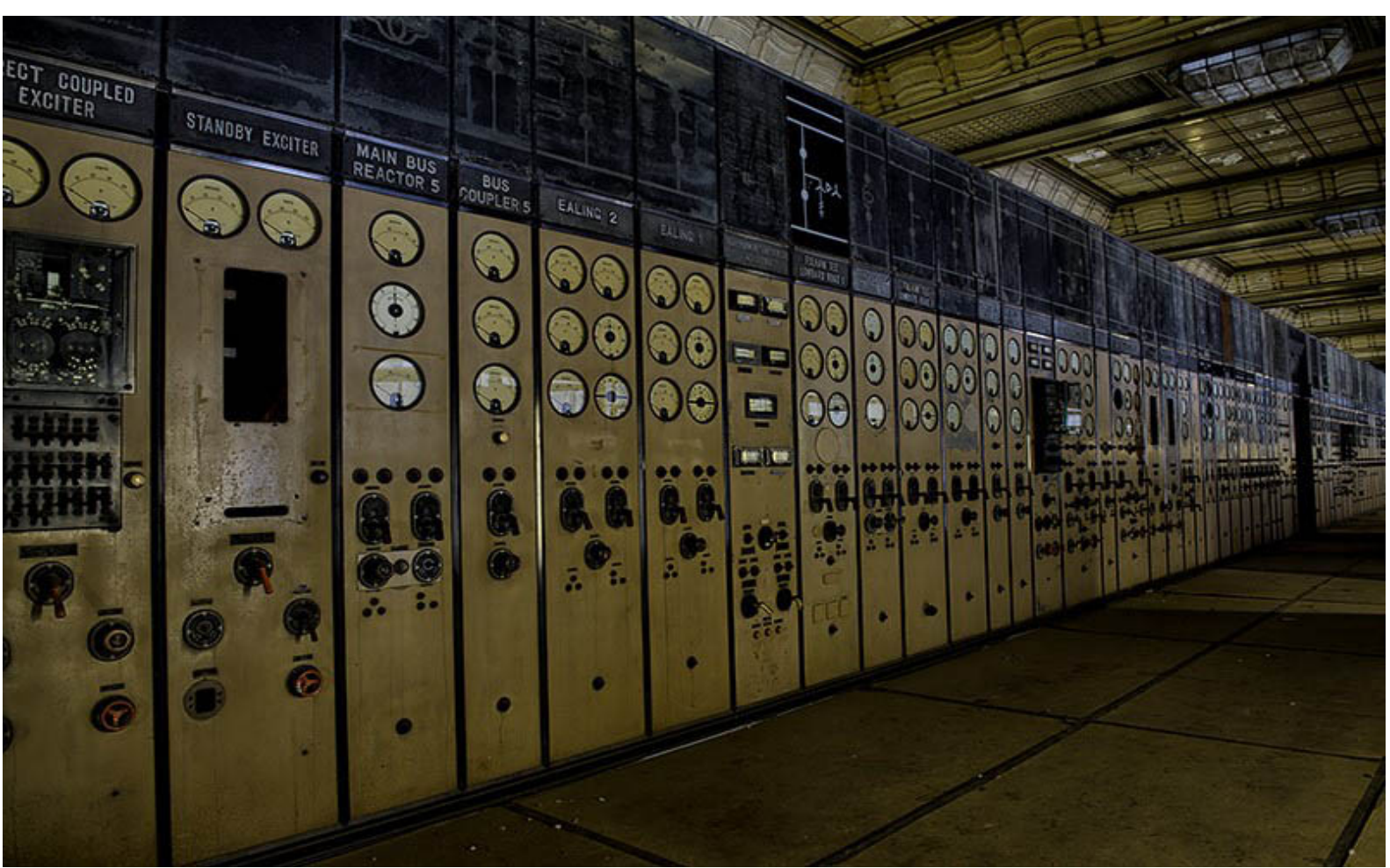

Figura 4. La sala di controllo della centrale, nonostante l'abbandono si è conservata. Sono ancora presenti i soffitti vetrati progettati da Halliday.

parte delle maggiori autorità6. Nel primo progetto del 1927, l'ingegnere Pearce ${ }^{7}$ aveva previsto l'inserimento di 16 ciminiere di ridotte dimensioni, 8 per ciascuna delle due unità, tutte ubicate lungo l'area centrale della costruzione. Tale particolare fu al centro di una lunga discussione pubblica i cui attori (governo della città e di quartiere, cittadini e rappresentanti della LPC) tentarono una mediazione, costringendo Pearce a ridurre il numero di ciminiere dalle 16 della prima idea progettuale, in 8, poi in 6 e, infine in 4 singole ciminiere, poste agli angoli della costruzione ${ }^{8}$. La riduzione del numero delle ciminiere però, ne causò l'aumento dimensionale, arrivando a prefigurare una costruzione dall'ancora maggiore impatto dimensionale e dalla forte caratterizzazione iconica. Nonostante i dialoghi in corso con l'amministrazione cittadina e con i numerosi detrattori della struttura, i problemi legati alla realizzazione della BPS, nel 1929 non erano definitivamente risolti. La dimensione della ciminiere rappresentava per molti una minaccia e una sicura fonte di inquinamento a cui Pearce decise di rispondere in modo definitivo tramite un progetto tecnologico e innovativo mai sperimentato prima, basato sul lavaggio dei gasi di scarico9. Per testare l'innovativo sistema, Pearce, realizzò una serie di sperimenti e di prototipi, grazie ai quali si assicurò della bontà della soluzione prevista che venne poi applicata in Battersea, eliminando molte della preoccupazioni iniziali10 (costo 2 mln pound secondo la LPC).

\footnotetext{
${ }^{6}$ Appare importante segnalare come nelle prime fasi del dibattito inerente la costruzione della centrale, quasi tutta l'opinione pubblica si scagliò contro il progetto, principalmente per le enormi dimensioni dell'intervento e per i possibili danni causati dall'inquinamento nella aree limitrofe.
}

${ }^{7}$ Standen Leonard Pearce (1873 - 1947) è stato un ingegnere elettrico e principale responsabile del progetto per la BAttersea Power Station. Il progetto impiantistico e distributivo in pianta venne affidato a lui poichè era impiegato presso la LPC vero la fine degli anni Venti. Fu autore di numerosi progetti in merito alla centrale, cercando di adeguare il disegno complessivo dell'impianto industriale alle richieste della cittadinanza e della municipalità, e fu responsabile dei disegni dei sistemi di lavaggio dei gas di scarico, utili a pulire le emissioni e a rendere la centrale meno inquinante.

8 I cambiamenti nel progetto seguirono le indicazioni della municipalità e dei cittadini, ricercando una mediazione fra le necessità della proprietà e le richieste degli altri attori coinvolti.

9 Vedi note precedenti. I progetti del gas washing system un tempo secretati per mantenere inviolata l'originalità del progetto, sono oggi conservati presso i National Archives di Londra.

10 Il costo della sperimentazione e della applicazione di tale sistema fu di 2 milioni di sterline secondo la LPC. 


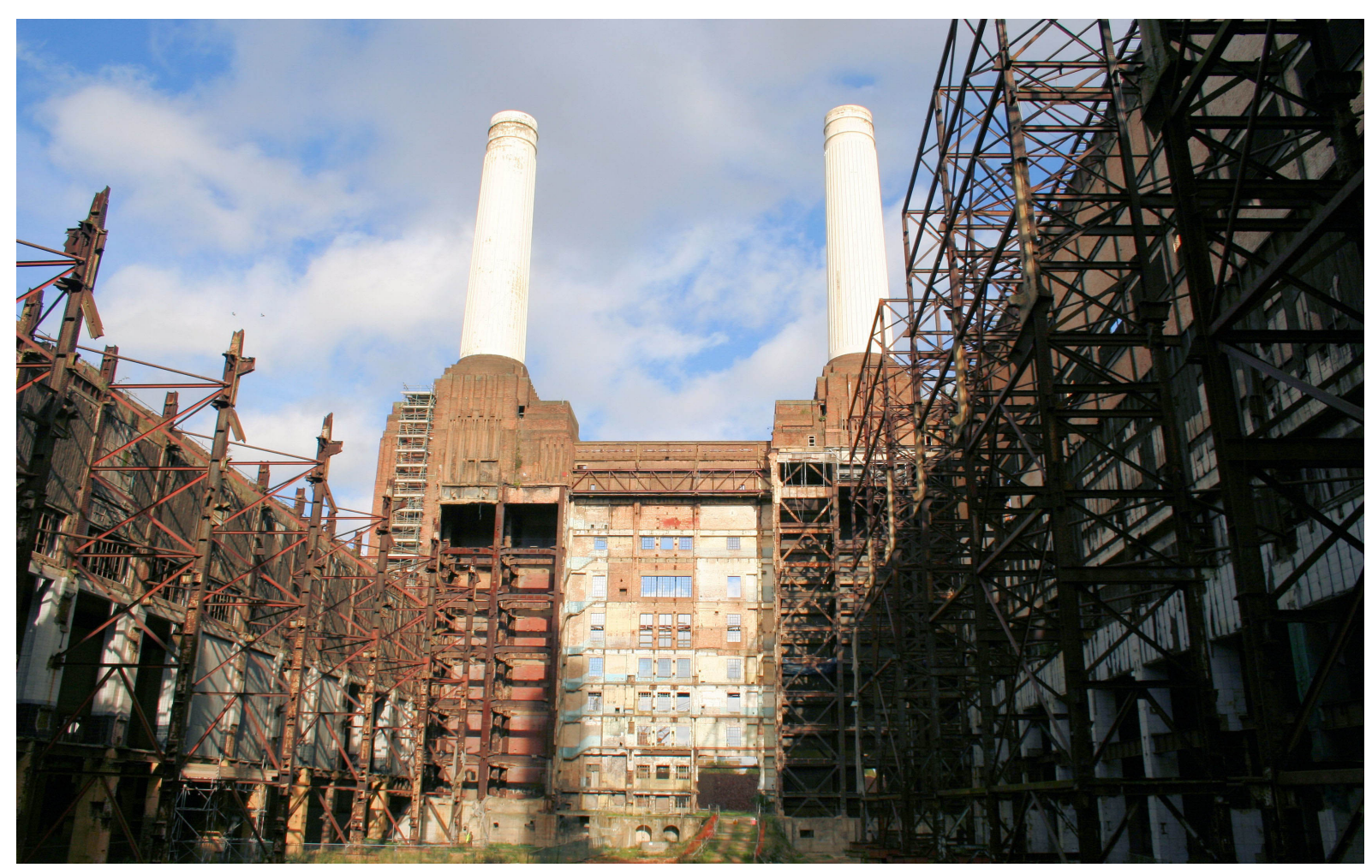

Figura 5. Lo spazio vuoto all'interno della centrale.

Il sistema così previsto, basandosi su di una serie di lavaggi dei gas di scarico, venne posizionato da Pearce alla base delle quattro ciminiere della BPS, andando a modificare un impianto planimetrico fino a quel momento più regolare. Da questi inserimenti, dipende, infatti, l'ampliamento dei volumi alla base delle quattro ciminiere, che vennero ingranditi rispetto alle versioni precedenti del progetto, allo scopo di ospitare i nuovi macchinari utili al lavaggio dei fumi di scarico ${ }^{11 .}$

Risolti i problemi legati all'inquinamento, la BPS esigeva una migliore attenzione verso l'architettura dell'esterno, al fine di creare un dialogo con la città e di poter così rappresentare al meglio il simbolo di un nuovo corso di sviluppo tecnologico e industriale. A tale scopo, a collaborare con Pearce venne chiamato uno dei maggiori architetti del periodo, ultimo discepolo della più importante famiglia di architetti inglesi: Giles Gilbert Scott 12 .

Nonostante quindi il piano per BPS fosse in larga parte ormai definito, il coinvolgimento di Scott rappresenta la significativa volontà da parte della committenza di creare non solo un edificio funzionale e poco inquinante, ma anche iconico, suggestivo, caratterizzante ${ }^{13}$. L'apporto di Scott, per sua stessa ammissione, riguarda solo l'aspetto esteriore dell'edificio. Come scrive in una lettera del 1947 circa il suo lavoro nella progettazione di centrali elettriche:

I confine my work entirely to matters of apparence. I prepare elevations, and when these are approved I do scale details and full-sizes, select the materials, visit the job occasionally to see that these materials are used in the right way, and inspect sample walling, etc. but I do not superintend the elevation, nor transact the business side. All this is done by

11 L'idea direalizzare volumi di maggiori dimensioni aglòi angoli della centrale trovò poi conferma anche negli elaborati di Scott per i prospetti esterni negli anni successivi.

12 Giles Gilbert Scott (1880-1960) architetto, è l'erende della più importante famiglia di architetti inglesi. Ottenne l'incarico per i prospetti esterni della centrale BSP giovanissimo, ritrovandosi da studente tirocinante a progettista di uno dei più grandi edifici della capitale. A tal proposito gli venne affiancato, nel primo periodo, un architetto anziano, suo mentore negli anni di tirocinio.

13 L'idea di coinvolgere un nome particolarmente noto allo scopo di mettere tutto d'accordo sulle future operazioni pare essere una tendenza costante nella storia della BPS, tendenza che si ripeterà numerose volte anche negli anni a venire. 
promoters' architectural staff, or other architect, who also prepare necessary working drawings embodying, of course, my details in them ${ }^{14}$ (Watts, 2016).

Il contributo di Scott, quindi, non è di carattere operativo o cantieristico ma piuttosto di natura formale in una duplice valenza. Da un lato, viene chiesto all'architetto inglese di occuparsi della forma della BPS, perchè l'anima produttiva, lo schema funzionale, gli spazi necessari sono già stati definiti dal team di ingegneri che opera per la LPC; dall'altro la sua partecipazione ha la funzione di dare una forma nuova al tipo di intervento agli occhi degli ancora dubbiosi cittadini. La presenza nel team di lavoro di Scott spiega la volontà di non realizzare una semplice costruzione finalizzata alla produzione di energia come molte altre presenti sul territorio londinese, ma piuttosto di costruire qualcosa di unico, di innovativo: una cattedrale dell'elettricità15. Questo coinvolgimento cambierà da subito l'idea stessa della costruzione che smetterà di rivestire il ruolo di edificio industriale dalle enormi dimensioni, per divenire un simbolo del cambiamento in atto a Londra. Lo sviluppo tecnologico, l'innovativa morfologia, la posizione centrale, le dimensioni inaudite, la firma di un prestigioso architetto, collaborano alla creazione di un edificio iconico che entrerà nel paesaggio urbano della capitale, cambiandola per sempre.

Scott, accettando l'incarico della LPC, intendeva dimostrare che le "power stations can be fine buildings"16 (Watts, 2016). Richiamando le parole di John Betjeman:

There is a tendency to classify power stations with factories and to locate them in industrial areas, but they differ from factories in one important respect: they are public utility undertakings, marketing their product in a localised and centralised market and the nearer they are to that market the more economically and efficiently they function ${ }^{17}$ (Watts, 2016).

Scott decise quindi per un progetto che sapesse elevare la centrale da comune fabbrica anonima in una vera e propria cattedrale dell'energia elettrica e del progresso. Così come avvenne per la cattedrale di Liverpool e per qualunque cattedrale in genere, infatti, la centrale di Battersea presentava una sala turbine che esigeva molto spazio in altezza, senza alcun orizzontamento all'interno di essa. Si presentava come uno spazio enorme, apparentemente vuoto o occupato solamente dalle turbine nella parte inferiore, e poteva quindi essere inteso architettonicamente e volumetricamente come la navata centrale di una cattedrale. Questa prerogativa consentì a Scott di concentrarsi unicamente sui prospetti dell'edificio, senza preoccuparsi dell'adeguamento funzionale degli spazi o dei collegamenti verticali, producendo elevazioni che potessero tendere alla ricerca di quel gusto estetico e stilistico per cui era stato coinvolto nel progetto. Adeguandosi ad altri progetti da lui realizzati e alla tradizione costruttiva londinese, progetto una struttura in ferro che servisse da scheletro all'edificio e ne ipotizzò un rivestimento in mattoni. La scelta di una superficie esterna in mattoni risultava particolarmente adeguata sia dal punto di vista economico/funzionale, sia dal punto di vista di inserimento all'interno del contesto cittadino: ricorrere a tale soluzione significava applicare una metodologia costruttiva tradizionale, normalmente presente in molti edifici pubblici e privati, da cattedrali, chiese a warehouses o magazzini portuali, ad un edificio che chiedeva di essere inserito a pieno titolo all'interno del paesaggio urbano della città, puntando a divenirne presto uno dei punti di riferimento architettonici e stilistici. Lo scavo delle fondazioni dell'edificio iniziò nel 1929, e presto ci si dedicò all'armatura di acciaio che avrebbe assolto la funzione di scheletro portante della struttura. La posa del rivestimento in mattoni, iniziò nel 1931 e proseguì fino al 1933, anno in cui si incominciò l'impegnativo lavoro di costruzione delle ciminiere. realizzazione di ciminiere con una tecnica innovativa detta "ferrocrete" costituita da una particolare amalgama di malta

\footnotetext{
${ }^{14}$ Le parole di Scott sono estratte dal portfolio Temples of Power, riportato anche da WATTS Peter, Up in smoke. The failed dreams of Battersea power station, Paradise Road, London, 2016, p. 52.

15 Il riferimento "cattedrale dell'elettricità" richiama gli appunti di Scott e molti articoli della critica architettonica che hanno visto tale finalità nel disegno della BPS da parte di Scott.

16 Peter Watts. Up in smoke. The failed dreams of Battersea power station, Paradise Road, London, 2016, p. 87.

17 idem.
}

(C) Labor \& Engenho, Campinas [SP] Brasil, v.11, n.4, p.477-491, out./dez. 2017.

DOI: http://dx.doi.org/10.20396/labore.v11i4.8651202 
cementizia rinforzata da una maglia metallica a rete elicoidale18. Inoltre, ancora una volta attento all'impatto del bene sulla città, Scott propose la tinteggiatura delle quattro ciminiere in colore ocra, cromia che meglio si sposava con i toni dei mattoni del rivestimento esterno ${ }^{19}$. Oltre all'attività e all'attenzione che Scott ha impiegato per il progetto e la realizzazione dei prospetti esterni, va segnalata la medesima considerazione per l'interno della BPS20. Tale aspetto non è di minore importanza: la volontà di creare un edificio unico e iconico doveva riguardare non solo la facciata esterna del bene, ma anche gli spazi interni, sebbene questi ultimi sarebbero stati inaccessibili alla città. La centrale, ormai completata dal punto di vista architettonico era pronta non solo ad entrare in funzione e a provvedere al sostentamento elettrico del centro della capitale, ma anche a divenire un nuovo simbolo della città, entrando a pieno diritto nello skyline del centro inglese.

\section{La BPS nell'immaginario collettivo}

L'importanza dimensionale, storica e produttiva della Battersea Power Station è facilmente documentabile, ma nel tempo, l'edificio ha assunto anche una notevole importanza figurativa e di vero e proprio simbolo/landmark urbano. In tale aspetto risiede una delle caratteristiche più affascinanti della storia della struttura che, dopo essere stata palesemente respinta dalle amministrazioni cittadine, è stata gradualmente accettata dai londinesi, fino a diventare non solo simbolo della città, ma anche del suo passato industriale. L'immagine della centrale è accettata e anzi difesa dalla cittadinanza inglese, sin dagli anni del dopoguerra. L'episodio più significativo, e che ha regalato alla BPS una enorme notorietà anche al di fuori dei confini nazionali, risale sicuramente alla scelta da parte di Roger Waters di usare una fotografia della centrale per la cover dell'album Animals del 197721. La scelta dell'edificio presentava numerose ragioni di carattere simbolico e politico, ma, principalmente, fu scelta la centrale per il suo grande impatto iconografico. Negli anni successivi, molte altre pellicole cinematografiche e non scelsero la centrale come set per le riprese di film a carattere fantascientifico o surreale, a riprova del grande impatto che l'edificio aveva nell'immaginario collettivo22. Perfettamente inserito nello skyline di Londra, infatti, rappresentava, già dagli anni '70, un esempio del passato industriale della città, ormai votata a più innovativi sistemi di produzione dell'energia 23 .

Proprio a seguito della arretratezza dell'impianto, ne fu deciso lo spegnimento nel 1977 e 198324 , ma non prima che le istituzioni si muovessero per la salvaguardia del bene e la sua tutela. Allo scopo di evitare, come era accaduto ad altre centrali londinesi, la ormai possibile demolizione di questo bene culturale della nazione, il Segretario di Stato per le politiche ambientali Micheal Heseltine, il 14 ottobre del 1980 insignì la Battersea Power Station del titolo di listed building di II grado25, assegnando quindi un vincolo strutturale all'edificio preservandolo per sempre da possibili attacchi speculativi26. Se sorprende l'assegnazione di un così prestigioso vincolo ad un

\footnotetext{
18 Valentina Corvigno. "Another brick out of the wall. Paesaggio urbano e autenticità materica nel destino della Battersea Power Station", in Ananke n.72 maggio 2014, pp. 120-127.

${ }^{19}$ L'originario color ocra venne poi rimpiazzato negli anni successicivi alla seconda guerra mondiale con il più noto color bianco.

${ }^{20}$ A tale scopo fu affidato il progetto degli interni all'architetto James Theodore Halliday che cooperò con Pearce alla definizione di alcuni problemi strutturali legati agli impianti interni e, principalmente, coordinò il disegno e il progetto delle sale di Controllo alla centrale, definendone progetto funzionale e aspetto estetico.

21 Pink Floyd, Animlas, pubblicato il 23 gennaio 1977. L'immagine di copertina venne scattata, dopo numerosi tentativi, e ritrae un maiale gonfiabile gigante che vola fra le ciminiere della BPS.

22 Fra i molti progetti cinematografici che hanno coinvolto la BPS si ricordano: Monty Python - Il senso della vita dei Monty Phyton del 1983, I figli degli uomini di Alfonso Cuaron del 2006, L'illusionista di Sylvain Chomet del 2010.

${ }^{23}$ Annie Graham \& Steve Graham. Battersea Power Station. An icon of our times, The Book Guild, Leics, 2015.

24 Venne spenta definitivamente prima la centrale A e poi la B.

25 L'elezione al vincolo è presente all'indirizzo http://historicengland.org.uk/listing/the-list/list-entry/1357620

${ }^{26}$ Steve Birney. The colossus of Battersea: a report by SAVE Britains's heritage, SAVE Britain Heritage, London, 1981.
} 
edificio che è ancora in funzione e sta ancora producendo energia elettrica, anche se solo per metà della propria estensione 27 , appaiono ancora più significative le motivazioni del vincolo:

It was added to Nation Heritage List of England in recognition of its powerful scale, celebrated silhouette, and that, as a power station it was the first to rationalise large-scale distribution of power. The building it's a masterpiece of industrial design. It's one of London's most prominent landmarks and one of a few with a genuine claim to the title iconic ${ }^{28}$.

Tale azione influì naturalmente in modo profondo nelle future trasformazioni della centrale, da un lato fu impedita la demolizione del bene, preservandolo da eventi meramente speculativi, dall'altro creò un forte freno alle volontà di investire nell'area, trasformando apparentemente la centrale da risorsa a criticità agli occhi degli investitori. La BPS, nei primi anni Ottanta e per i venti anni successivi, attraversa un momento cruciale per il proprio destino. L'edificio della centrale elettrica estremamente importante per l'architettura, la storia e la cultura industriale della città, viene spesso utilizzato in pellicole cinematografiche, spot pubblicitari, campagne fotografiche, perché il suo impatto iconico è unico nel panorama inglese, mentre la sua materia deperisce gradualmente, totalmente priva di ogni intervento di conservazione o restauro. Nello stesso periodo però, vengono ipotizzati molti progetti per la sua trasformazione funzionale, senza che nessuno convinca gli imprenditori locali, l'amministrazione cittadina o il popolo londinese, restando ipotesi di intervento e non venendo mai applicate.

\section{Ipotesi di trasformazione}

Dai primi anni Ottanta, ai primi anni dieci del secolo successivo, moltissimi imprenditori investirono nel progetto di rifunzionalizzazione dell'area della BPS, avanzando ciascuno una nuova filosofia per l'intervento ${ }^{29}$ (Watts, 2016).

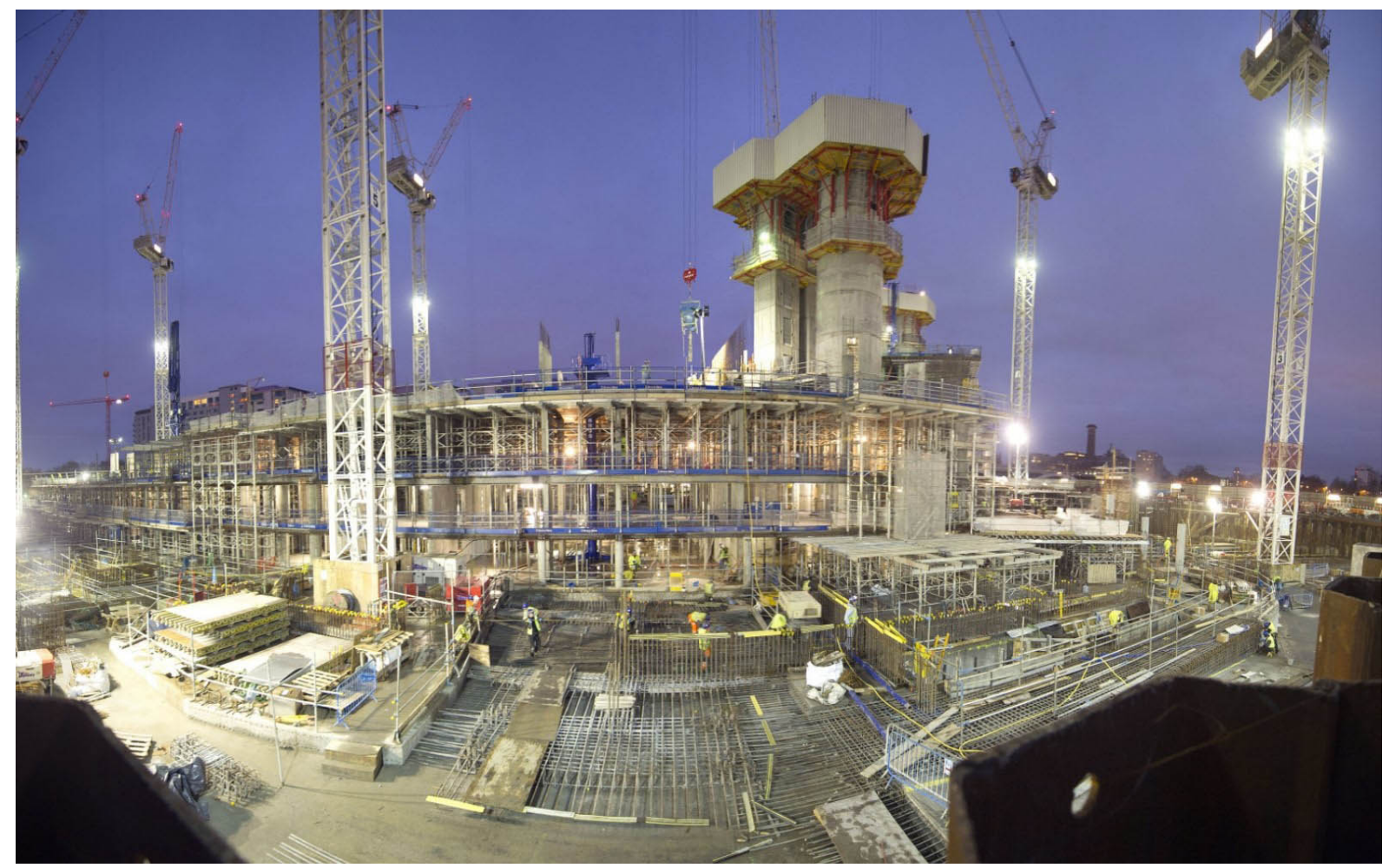

Figura 6. Il cantiere per la demolizione e ricostruzione delle ciminiere.

\footnotetext{
${ }^{27} \mathrm{Nel} \mathrm{momento} \mathrm{del} \mathrm{riconoscimento} \mathrm{a} \mathrm{listed} \mathrm{building} \mathrm{è} \mathrm{in} \mathrm{funzione} \mathrm{solo} \mathrm{la} \mathrm{centrale} \mathrm{B,} \mathrm{mentre} \mathrm{la} \mathrm{A} \mathrm{è} \mathrm{stata} \mathrm{dismessa} \mathrm{pochi}$ anni prima.

28 idem.

${ }^{29}$ Per riassumere il complesso periodo di trasformazioni si rimanda a Peter Watts, Up in smoke. The failed dreams of Battersea power station, Paradise Road, London, 2016.
} 
Appare interessante sottolineare come tale tendenza, riassuma in modo molto efficace ciò che è stata la percezione dei beni culturali in Inghilterra nelle ultime decadi. I primi progetti, votati ad una attenta conservazione della centrale e caratterizzati da destinazioni d'uso pubbliche e pensate per i cittadini, si è gradualmente assistito a progetti più coraggiosi, pensati con attenzione alle ragioni della speculazione edilizia e del massimo profitto.

La prima destinazione d'uso che apparve versoimile per l'area fu avanzata da un ingegnere irlandese Mark Leslie, che ipotizzò di collocare all'interno della centrale un museo dell'industrializzazion ${ }^{30}$. Volendo enfatizzare l'importanza dell'edificio nell'evoluzione produttiva e industriale di Londra, ipotizzò un museo diviso in aree distinte che volevano riassumere le fasi dell'industrializzazione (vapore, elettricità) e il futuro della produzione. Tale progetto si poneva in modo totalmente armonioso rispetto al bene disegnato da Scott, in quanto le tre aree distinte trovavano spazio all'interno dei diversi corpi della centrale, e quindi non presupponevano massicce demolizioni o trasformazioni del bene, conservandolo pressochè nelle medesime condizioni in cui si trovava in seguito alla dismissione. Tale idea però, non potendosi avvalere delle necessarie risorse finanziarie, fu presto trasformata da uomini d'affari soci di Leslie ${ }^{31}$, nell'area della BPS, di un grande parco divertimenti per famiglie. Questo gruppo si costituì in forma ufficiale e, dopo aver manifestato pubblicamente il proprio interesse, acquistò il lotto e la centrale BPS, dichiarando da subito la propria volontà di realizzare un parco divertimenti per famiglie in quella stessa area. John Broome si presentò come capofila e investitore unico in questa impresa ${ }^{32} \mathrm{e}$ intraprese una assidua campagna pubblicitaria per la realizzazione del parco, che, secondo le previsioni, avrebbe aperto al pubblico nel 1990. Il parco divertimenti, nonostante i progetti e le coraggiose dichiarazioni di Broome alla stampa, non fu mai realizzato33, e l'area cambiò proprietario nel 1993, passando alla società Parkview della famiglia Hwang. Lungo i tredici anni successivi, i nuovi proprietari si impegnarono nella realizzazione di numerosi progetti per il sito, aumentandone anche le dimensioni passando dai 18 acri iniziali ai 36 del 200634. Nonostante gli sforzi profusi e la seria intenzione di realizzare in questa zona di Londra un progetto misto commerciale e residenziale, in questo lungo periodo furono intraprese solo alcune opere di conservazione alla struttura, oramai abbandonata e priva di ogni forma di manutenzione 35 , ma non si avanzò alcuna operazione di trasformazione per il sito. Destino analogo riguardò la Trasury holdings, che acquistò l'area nel 2006 e che non realizzò alcuna nuova struttura nell'area ma si limitò a commissionare progetti complessivi e ipotesi di intervento.

I nuovi proprietari, emulando quanto succcesso negli anni della costruzione della centrale, decisero di rivolgersi ad un importante architetto internazionale, sperando che l'importanza del nome coinvolto potesse convincere l'amministrazione cittadina e i londinesi della bontà del progetto. Fu quindi affidato all'architetto uruguaiano Rafael Vinoly, il mandato di ipotizzare un masterplan di intervento per l'area, allo scopo di ubicare in essa nuovi edifici residenziali e trasformando l'antica centrale in un innovativo polo commerciale. Il masterplan fu presentato nel 2008 e colpì per l'azzardo di collocare, a ridosso della centrale una alta torre dal design contemporaneo, fortemente caratterizzata da tecnologie di risparmio energetico. Il masterplan non ottenne l'approvazione dell'amministrazione cittadina, principalmente per l'audacia dei nuovi inserimenti che attirarono molte critiche ma furono funzionali a non attirare l'attenzione dei più sull'ambizioso piano di trasformazione del rudere della centrale in polo commerciale.

\footnotetext{
${ }^{30}$ Leslie, in realtà, rispose ad un bando pubblicato sui principali organi di stampa, finalizzato a sondare l'interesse e nuove idee per il futuro della centrale.

31 I soci di Leslie furono gli uomini d'affari inglesi David Roche e John Broome.

32 Broome con una abile campagna mediatica riuscì ad acquisire le quote dei soci, escludendoli gradualmente dal progetto e diventando così unico proprietario della centrale.

33 John Broome si indebitò fortemente nella ricerca di costruire il proprio parco divertimenti, senza però riuscire a portare a termine l'impresa e trovandosi così costretto a vedere la proprietà del sito a nuovi acquirenti asiatici.

34 I nuovi proprietari acquisirono molte aree dismesse limitrofe al sito della BPS, aumentando così la capacità edificatoria dell'area.
}

35 Durante l'era Broome, la centrale ha perso la copertura originaria, crollata e parte del muro ovest, trasformandosi in uno scheletro senza alcuna funzione possibile senza inmportanti interventi di ricostruzione o completamento. 


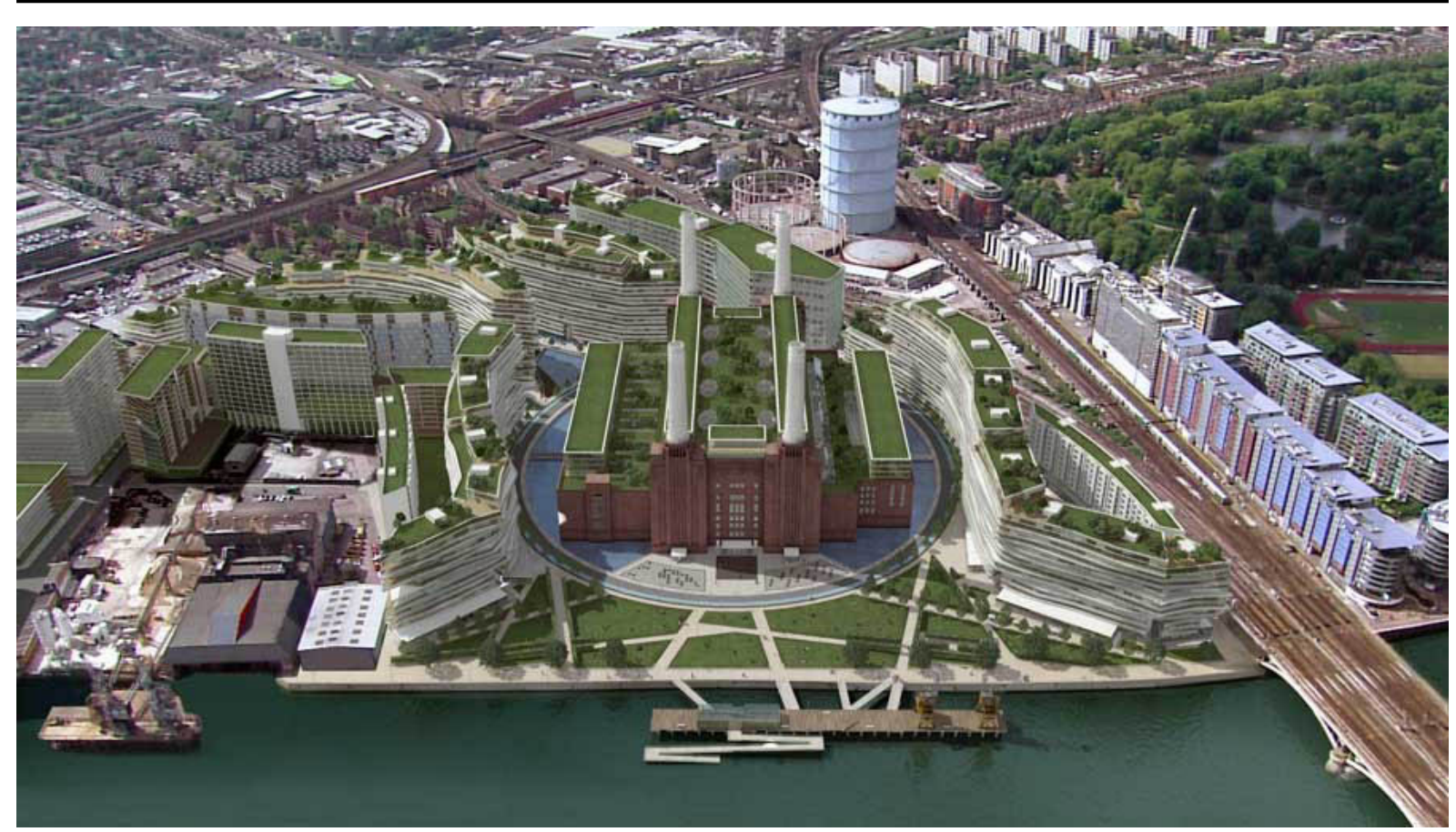

Figura 7. Rendering aereo del progetto in corso di realizzazione. Fonte: http://batterseapowerstation.co.uk/

Vinoly presentò un secondo masterplan nel 2010 che, sostanzialmente riprendeva i concetti principali del primo (residenza + commercio), eliminando però l'impatto dell'alta torre, sostituendola con edifici più bassi, la cui altezza massima era la stessa del corpo centrale della BPS. L'intervento sull'edificio rimaneva sostanzialmenteanalogo al precedente, ricercando la conservazione dello scheletro e l'ubicazione all'interno di un nuovo polo commerciale. Tale masterplan, risalente al 2010, ottenne l'approvazione della municipalità e, anche grazie al subentro dei nuovi proprietari malesi della SP Setia, i lavori iniziarono poco dopo.

\section{Nuovo masterplan, nuovo futuro, antiche icone.}

Il nuovo masterplan, prevede la realizzazione di differenti lotti di edifici residenziali, ubicati nelle aree adiacenti la centrale e un tempo destinate allo stoccaggio della materia prima o alla sua movimentazione. Il progetto globale prevede la realizzazione di un nuovo grande polo residenziale per una utenza di alto livello, al cui centro trova spazio l'antica centrale, convertita a nuovo centro commerciale.

I diversi lotti di costruzione, tutti di nuova realizzazione e a destinazione residenziale, sono stati affidati a studi di architettura di importanza internazionale, garantendo a ciascuno la libertà di poter restare fedeli alle proprie inclinazioni stilistiche e progettuali, rispettando solamente alcune indicazioni sulle volumetrie da rispettare, il dimensionamento, la destinazione d'uso e le altezze massime. Per rendere l'area maggiormente funzionale e fruibile sono state inserite in progetto una nuova fermata della metropolitana cittadina e un nuovo punto di sosta per il trasporto fluviale sul Tamigi 36 . Il complesso architettonico, quindi, si presenta come una delle aree di maggior trasformazione a livello globale, richiedendo un investimento iniziale di alcuni miliardi di sterline.

Ciò che appare di sicuro interesse nell'ambito dell'intervento, oltre alla scelta, già vista in precedenza studiando la storia della centrale, di affidare l'incarico di ogni singolo lotto a studi di architetti estremamente celebri (Norman Foster, Frank 0. Gehry, ...) è il ruolo della Battersea Power Station all'interno del progetto. Analizzando i comunicati stampa e la presentazione dell'intervento alla cittadinanza, il logo del masterplan, le pagine internet ufficiali, risulta evidente come la centrale sia stata utilizzata in quanto è la migliore presentazione possibile per il masterplan e il progetto. La volontà di usare l'immagine consolidata della centrale nell'immaginario collettivo è infatti,

${ }^{36}$ Questi nuovi collegamenti saranno realizzati nell'ultima fase del masteplan, nel 2020.

(C) Labor \& Engenho, Campinas [SP] Brasil, v.11, n.4, p.477-491, out./dez. 2017. 

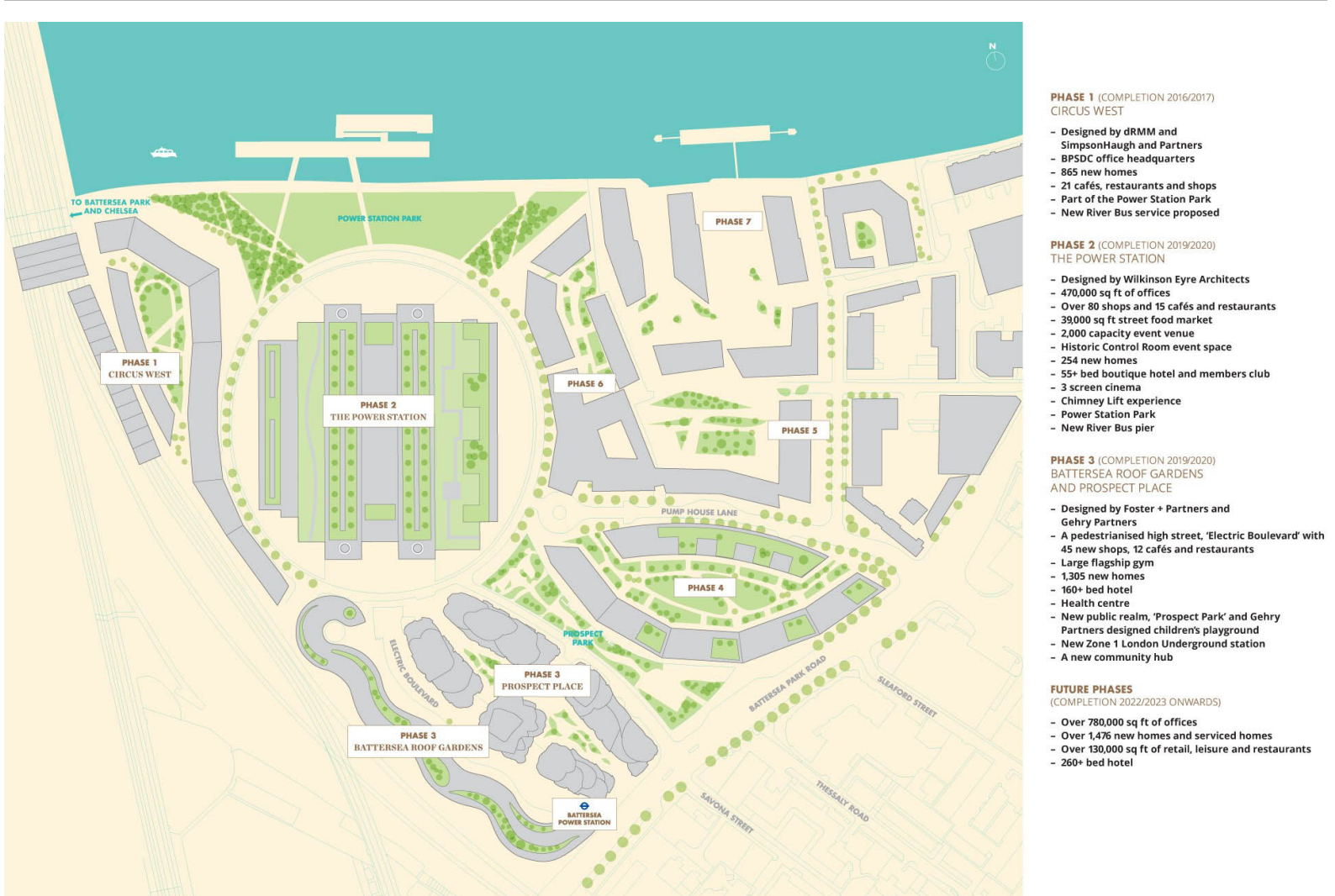

Figura 8. Mappa complessiva dell'intervento con la suddivisione in lotti. Fonte: http://batterseapowerstation.co.uk/

comprensibile se si riflette sulle ragioni speculative e commerciali di un progetto edilizio che espone così tanto gli investitori, ma risulta di difficile condivisione se analizzato in rapporto al passato. Sembra infatti che con l'intervento in corso, la cui conclusione si ipotizza sarà nel 2020, si sia raggiunta una nuova forma di trasformazione e di utilizzo della centrale. I primi anni successivi all'abbandono erano stati caratterizzati da idee di rifunzionalizzazione votate al sociale come musei dell'industria o centri sportivi, ma dalla sostanziale mancanza di potere economico per realizzare tali investimenti, gli ultimi anni del secolo scorso erano stati caratterizzati da nuovi proprietari con ottime possibilità di investimento che non hanno mai ottenuto però l'autorizzazione da parte della municipalità e dei cittadini a trasformare per fini speculativi la centrale di Battersea e che si sono dovuti accontentare di sfruttare il potere iconico della centrale, utilizzandola anche come quinta per la proiezione di immagini pubblicitarie ${ }^{37}$.

Figura 9. La "nuova" BPS fagocitata dai nuovi interventi. Fonte: http://batterseapowerstation.co.uk/

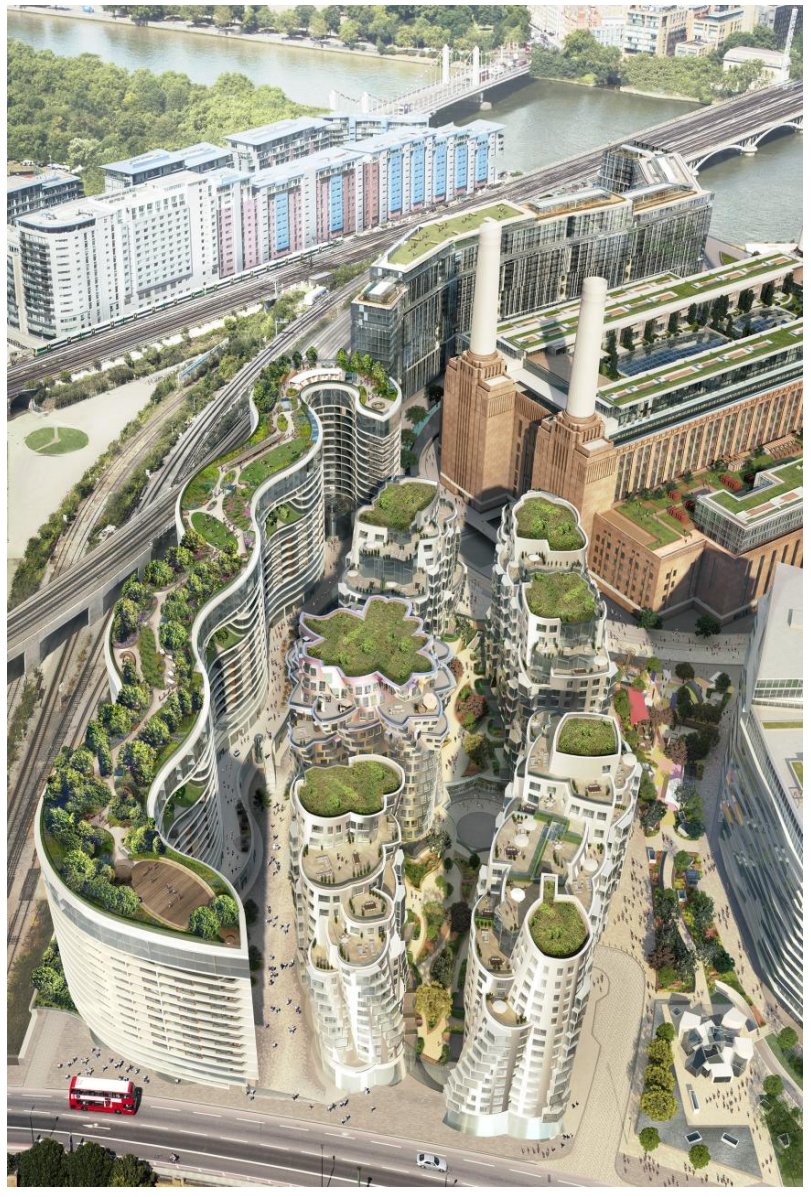

37 Ciò è avvenuto durante la proprietà Hwang, che ha pensato di proiettare alcune immagini pubblicitarie sui muri del rudere della centrale, alla metà degli anni 90. 


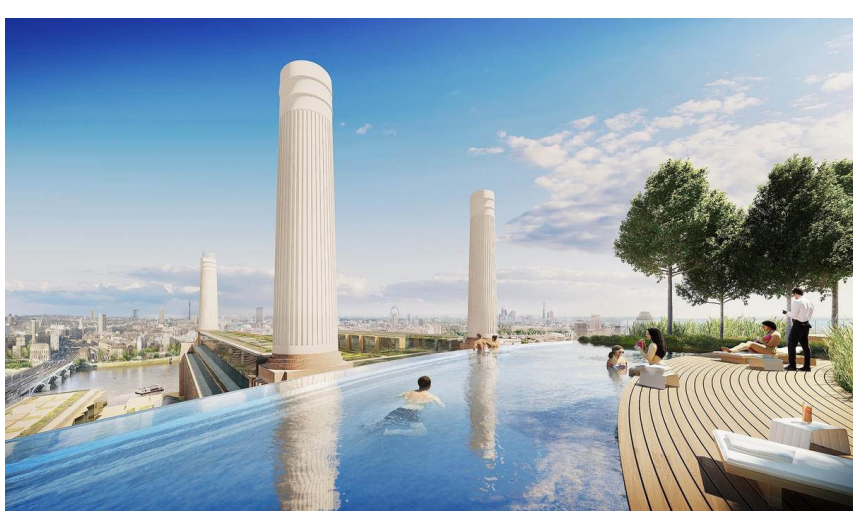

Figura 10. Rendering della BPS dal rooftop garden ddel lotto numero 2 in fase di ultimazione.

Fonte: http://batterseapowerstation.co.uk/

Nell'ultima fase, contemporanea, sembra possibile ipotizzare come le due istanze, quella economica e quella conservativa, abbiano trovato un sostanziale accordo che però privilegia la prima rispetto alla seconda. La motivazione del nuovo masterplan, infatti, votandosi radicalmente al residenziale e al commerciale, evidenzia come la comprensibile esigenza dei nuovi proprietari sia la massimizzazione di un profitto orami certo nella città che da alcuni anni detiene il primato per il costo più alto al metro quadro per la residenza. L'esclusività del masterplan, denotata dalla firma di archistar e da prezzi inaccessibili se non alle tasche di pochissimi, spiega come le priorità dell'intervento siano di carattere economico e come il rudere della centrale sia stato trasformato da enorme criticità in potenzialità perché viene presentato in quanto memoria di se stesso più che di rudere da conservare. Si potrebbe quindi affermare come l'immagine iconica della cattedrale dell'energia elettrica ha rappresentato quindi la ragione della sua conservazione e delle ragioni del vincolo, ma anche la sua più grande condanna, costringendo i nuovi proprietari a sfruttarne principalmente l'immagine, ma ignorandone le criticità materiche, conservative e architettoniche.

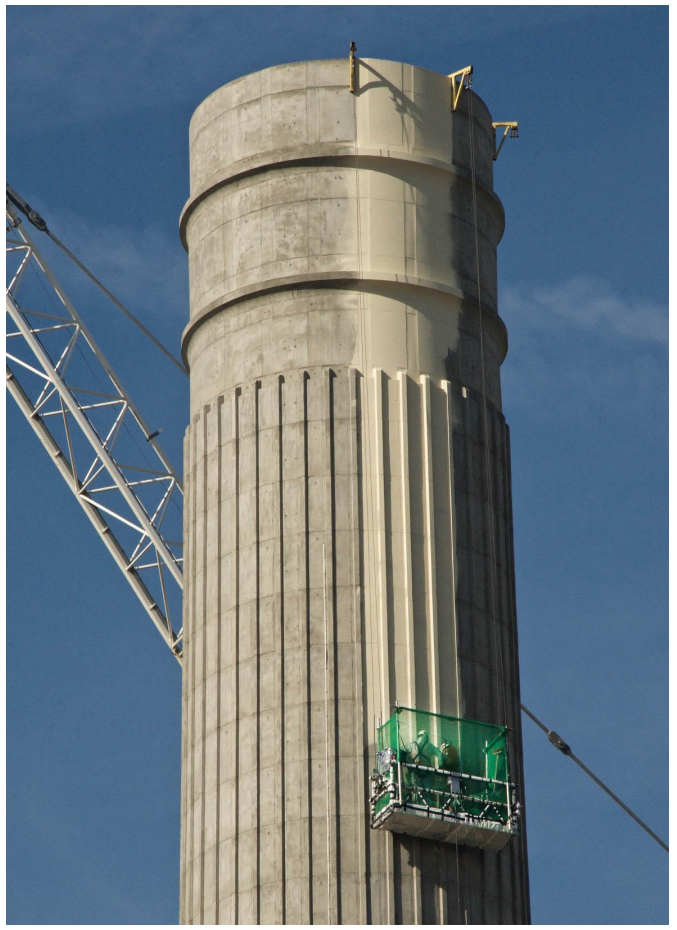

Figura 11. Ritinteggiatura delle ciminiere dopo la ricostruzione.

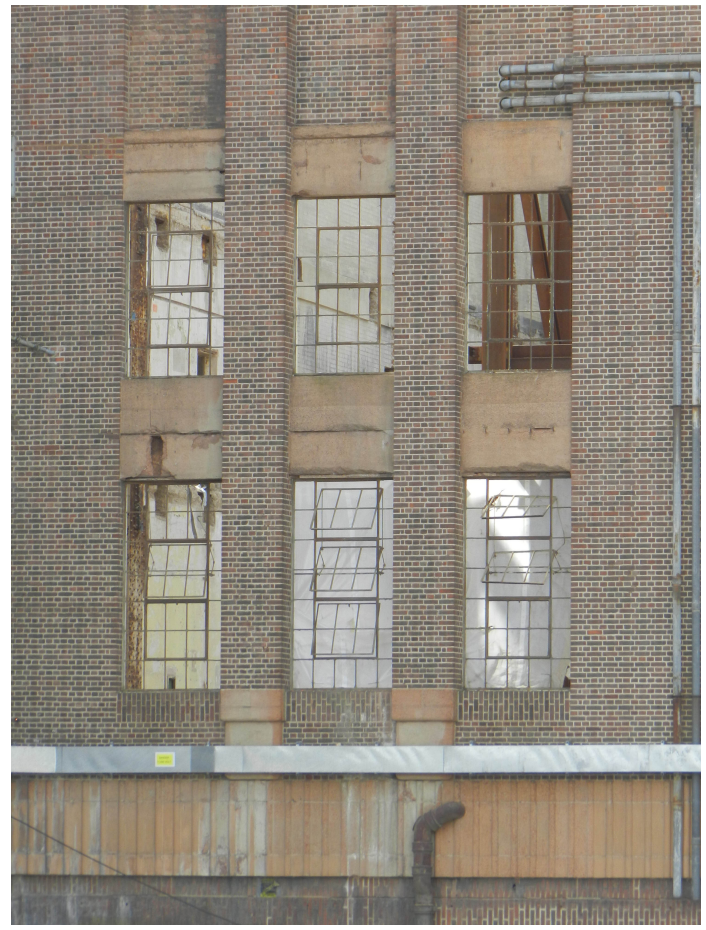

Figura 12. Particolare dei serramenti del prospetto Nord.

A sostenere tale ipotesi ci sono gli interventi di restauro già realizzati e molto ben documentati nella home page del sito38.

Sotto la voce Restoration, vengono infatti presentate le iniziative e le lavorazioni compiute principalmente sulle ciminiere dell'antica fabbriche che sono state, in sostanza, distrutte e ricostruite. Prive di alcuna funzione reale e vero simbolo della centrale, deteriorate dagli anni ma senza alcun pericolo di crollo reale, le ciminiere sono state distrutte e sostituite da altre, nuove, ma con una

38 Il masterplan è riccamente presentato nel sito http://batterseapowerstation.co.uk/ 
sezione interna più larga ${ }^{39}$ (Garner, 2008). Saggi esplorativi particolarmente approfonditi hanno potuto individuare l'esatto colore bianco che le ciminiere possedevano prima del degrado, colore che è stato scelto per la nuova tinteggiatura, ignorando le fasi successive alla costruzione in cui le ciminiere erano state tinteggiate di ocra, poi modificato dopo la seconda guerra mondiale nel bianco che è visibile anche oggi. Inoltre, la restoration della centrale, viene documentata anche attraverso le opere di manutenzione delle gru posizionate sulla riva del Tamigi, un tempo utili a raccogliere il coke che veniva portato alla centrale via fiume.
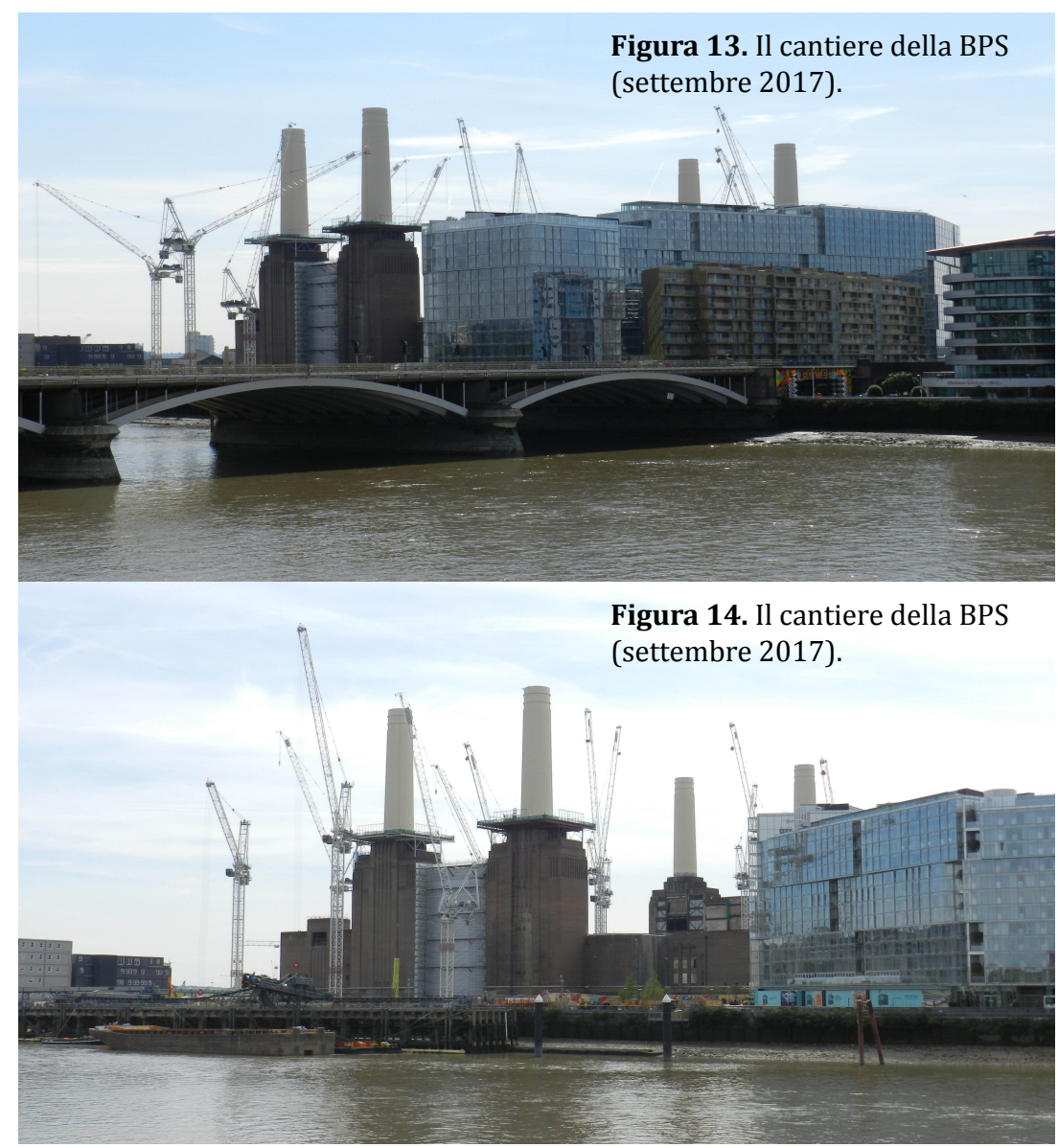

dell'elettricità. Si ipotizza una nuova copertura vetrata senza apparenti riferimenti alla precedente oggi crollata. Non sono altresì presenti indicazioni circa la sala controllo dell'antica BPS, riccamente decorata dall'attività di Halliday, così come non sono presenti cenni circa le porte bronzee di ingresso all'edificio, di cui si ignora l'attuale ubicazione. Resta quindi il piano delle ipotesi o la possibilità di attendere l'esito dei lavori che, nel frattempo, procedono spediti e hanno già ultimato (nel settembre del 2017) la demolizione e ricostruzione delle ciminiere della centrale.
Il nuovo progetto intende conservare le gru originarie in quanto memoria storia del passato industriale del bene, senza assegnare ad esse alcuna funzione, ma inglobandole in un parco residenziale che le trasformerà da simboli del passato industriale a cimeli storici decontestualizzati. Non è possibile però, all'interno della sezione Restoration del sito, conoscere l'entità degli interventi che il corpo della centrale affronterà per modificare la propria funzione in polo commercia-le. Il rudere della BPS, ridotto a scheletro dai decenni di abbandono, privo della copertura originaria, di ogni tipo di impianto interno, dei serramenti, di alcuni frammenti della muratura originaria, verrà radicalmente trasformato in un centro commerciale di più piani, perdendo naturalmente la caratteristica apparenza di cattedrale

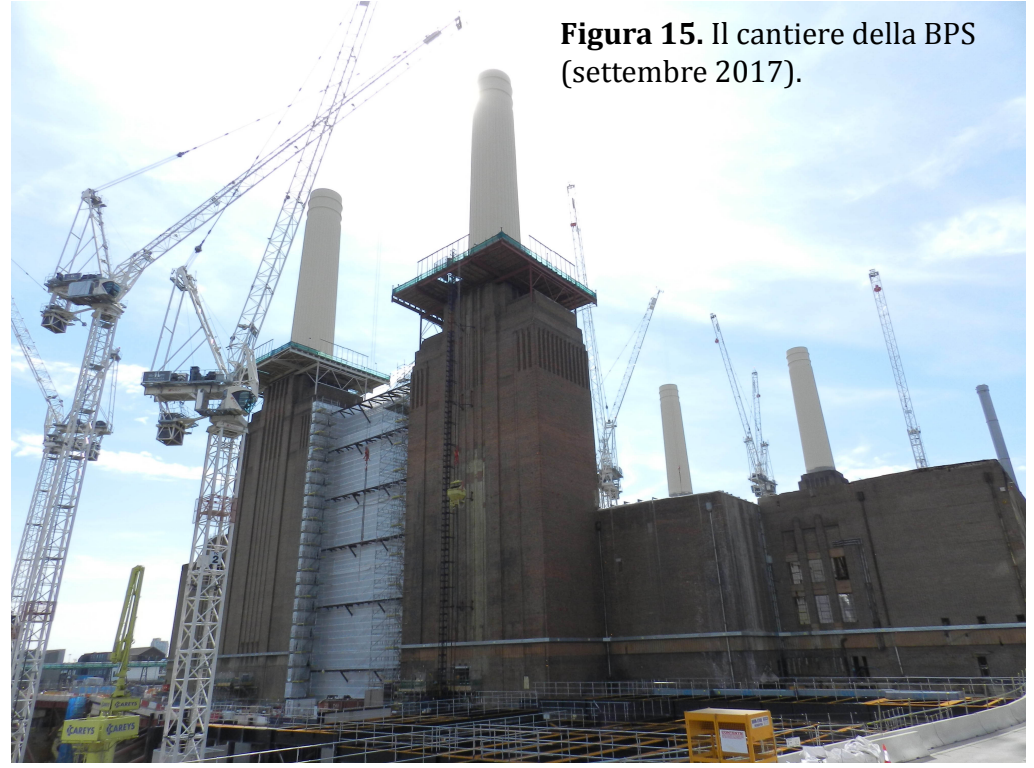

39 Keith Garner, "Battersea Power Station. An account of the proposed demolition and rebuilding of the chimneys", in Architectural Conservation Journal, vol. 14 n.2, luglio 2008, pp. 15-31. 
Emerge però molto più che un'ipotesi dallo studio delle trasformazioni in corso a Londra alla BPS: l'intenzione del progetto è stata soprattutto, sin dall'inizio, di restaurare l'immagine, l'icona della centrale, piuttosto che conservarne la materia e l'architettura. Nel passato dell'edificio, dal momento della sua dismissione, la centrale dell'elettricità ha vissuto un duplice destino: mentre la sua essenza materica, formale e architettonica deperiva, dimenticata da ogni proprietà che si sia alternata alla guida del progetto, la sua forza iconica cresceva, tanto da coinvolgere non solo i londinesi, ma anche i cittadini inglesi ed europei, diventando un simbolo senza tempo. Le trasformazioni in atto si sono concentrate sulla conservazione di questo bagaglio prestigioso, proseguendo sulla strada di elevare il simbolo sopra tutto, anche sacrificando la materia e l'autenticità.

\section{Referenze}

Birney, S. (1981). The colossus of Battersea: a report by SAVE Britains's heritage. London : SAVE Britain Heritage.

Corvigno, V. (2014). "Another brick out of the wall. Paesaggio urbano e autenticità materica nel destino della Battersea Power Station". Ananke n.72 maggio 2014, pp. 120-127.

Eletric Council (1987). Elettricity supply in the UK: A Chronology. s./l. : s./d.

Garner, K. (2008). "Battersea Power Station. An account of the proposed demolition and rebuilding of the chimneys". Architectural Conservation Journal, 14 (2) 15-31.

Graham, A., \& Graham, S. (2015). Battersea Power Station. An icon of our times. Leics : The Book Guild.

Horne, M. (2012). London area supply: a survey of London eletric lighting and power stations. s/l. : s/d.

Pedroche, B. (2013). London's lost Power Stations and Gasworks. s./l.. : The History press.

Sabbagh, K. (2000). Power into art: creating the Tate modern. s/l. : Bankside, Penguin Group.

Stirling, E. (1992). The history of the gas light \& Coke company 1812-1949. s./l. : A.\&C. Black.

Watts, P. (2016). Up in smoke. The failed dreams of Battersea power station. London : Paradise Road. 